\title{
Aplicación fraccionada de fertilizantes vía fertirriego y la eficiencia del nitrógeno, fósforo y potasio en calabacita
}

\author{
Split fertilization applied through fertigation and nitrogen phosphorus and potassium efficiency in squash \\ Daniela Alvarado-Camarillo ${ }^{1}$ (D), Luis Alonso Valdez-Aguilar ${ }^{2 *}(\mathbb{D}$, Juan Manuel Cepeda-Dovala1 (D), \\ Martín Rubí-Arriaga ${ }^{3}$ (D) Joel Pineda-Pineda ${ }^{4}$ (iD
}

${ }^{1}$ Departamento de Ciencias del Suelo, Universidad Autónoma Agraria Antonio Narro (UAAAN), Calzada Antonio Narro 1923, 25315, Saltillo, Coahuila, México ${ }^{2}$ Departamento de Horticultura, UAAAN. ${ }^{3}$ Facultad de Ciencias Agrícolas, Universidad Autónoma del Estado de México, 50200, El Cerrillo Piedras Blancas, Toluca, Estado de México, México. ${ }^{4}$ Departamento de Suelos, Universidad Autónoma Chapingo, Carretera México-Texcoco km 38.5, 56230, Texcoco, Estadode México, México. *Autor para correspondencia: luisalonso.valdez@uaaan.edu.mx

Fecha de recepción:

9 de abril de 2020

Fecha de aceptación:

1 de agosto de 2020

Publicado en línea:

19 de febrero de 2021

Este es un artículo en acceso abierto que se distribuye de acuerdo a los términos de la licencia Creative Commons.

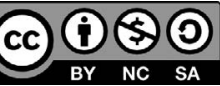

Reconocimiento-

NoComercial-

CompartirIgual 4.0

Internacional

\section{RESUMEN}

El fertirriego permite suplementar los fertilizantes en el momento y lugar requeridos, lo que aumenta la eficiencia en el uso de estos. En el presente estudio se determinó si la cantidad de fertilizantes minerales aplicados al cultivo de calabacita puede ser reducida, en comparación con la dosis regional recomendada, al fraccionar la aplicación a través de un sistema de fertirriego. Se diseñaron tres tratamientos: $100 \%$ de la dosis recomendada (120-33.6-174.3 kg ha-1 N-P-K), aplicada en forma sólida desde el momento de la siembra, y $62.5 \%$ y $50 \%$ de la dosis del tratamiento testigo, pero aplicados en forma fraccionada durante todo el ciclo del cultivo. Ni el rendimiento de fruto ni la concentración de N, P, Ca, y Mg en la parte aérea fueron afectados cuando la dosis se redujo a $62.5 \%$ o $50 \%$; sin embargo, las plantas mostraron mayor eficiencia en la recuperación del N y P, así como mayor eficiencia agronómica por unidad de N, P y K aplicados. Se concluye que la fertilización fraccionada aplicada a través del fertirriego permite reducir la dosis de fertilización sin afectar el rendimiento ni el crecimiento de calabacita debido a un aumento en la eficiencia de recuperación y agronómica de los nutrientes aplicados.

\section{PALABRAS Clave}

Agricultura sustentable, nutrición mineral, fertilización de cultivos, eficiencia agronómica, eficiencia de recuperación

\section{ABSTRACT}

Fertigation allows fertilizers to be supplemented at the required time and place, increasing their use efficiency. The present study determined whether the amount of mineral fertilizers applied to squash can be reduced, compared to the recommended dose, by fractioning their application through a fertigation system. Three treatments were designed: $100 \%$ of the recommended dose (120-33.6-174.3 $\mathrm{kg} \mathrm{ha}^{-1} \mathrm{~N}-\mathrm{P}-\mathrm{K}$ ) applied in solid form at sowing, and 62.5\% and 50\% of the control treatment, but with a split application through the growing season. Neither the fruit yield nor the concentration of $\mathrm{N}, \mathrm{P}, \mathrm{Ca}$, and $\mathrm{Mg}$ in the shoot were affected when the dose was reduced to $62.5 \%$ or $50 \%$; however, the plants showed greater efficiency in the recovery of $\mathrm{N}$ and $\mathrm{P}$ and greater agronomic efficiency per unit of N, P and K applied. In conclusion, fractionated fertilization applied through fertilgation reduces the fertilization dose without affecting the squash yield or growth due to an increase in both the recovery and agronomic efficiencies of the applied nutrients.

\section{KEYWORDS}

Sustainable agriculture, mineral nutrition, crop fertilization, recovery efficiency, agronomic efficiency 


\section{INTRODUCCIÓN}

La calabacita (Cucurbita pepo L.) es una hortaliza de gran relevancia agrícola y social en México. En 2019 se reportaron 30,000 ha cultivadas de esta especie, en las cuales se produjeron $615,000 \mathrm{t}$ de fruto, con un rendimiento promedio de $20.4 \mathrm{t} \mathrm{ha}^{-1}$ (SIAP 2019). México ocupa el sexto lugar mundial como país productor de calabacita y su producción representa $2.6 \%$ de la producción global (SIAP 2019). De esta hortaliza producida en el país, $84 \%$ es exportado a Estados Unidos, Japón y Canadá (SIAP 2019).

La aplicación excesiva de fertilizantes minerales (de síntesis química) en algunos casos es justificada por los agricultores ante eventos climáticos extremos, como es el caso de los huracanes, en los que la alta precipitación lixivia los nutrientes del suelo (Hendricks et al. 2019). Sin embargo, en general, una sobrefertilización de los suelos, así como una fertilización limitada, se consideran prácticas no deseables por las consecuencias negativas para el medio ambiente, así como por la reducida eficiencia económica (Sadeghpour et al. 2017), además del decremento de la biodiversidad y la degradación de los suelos (Díaz et al. 2016).

En la producción de calabacita, se emplean cantidades altas de fertilizantes minerales (Apáez-Barrios et al. 2019), especialmente el nitrógeno (N) (Chance III et al. 1999); por ello, en la actualidad, existe un fuerte interés por reducir el uso de fertilizantes minerales que afectan adversamente el medio ambiente (Díaz et al. 2016). Para lograrlo, en el caso de la calabacita, se han propuesto diferentes tecnologías; por ejemplo, la aplicación de zeolita en combinación con la fertilización mineral ha permitido reducir en $25 \%$ la cantidad de $\mathrm{N}$ y fósforo $(\mathrm{P})$ aplicados, luego de lo cual se ha reportado un rendimiento superior al de las plantas con la fertilización base (Apáez-Barrios et al. 2019). Otras tecnologías incluyen la sustitución parcial o total de los fertilizantes minerales por fertilizantes orgánicos o por biofertilizantes, como reportan Alvarado-Carrillo et al. (2018), quienes obtuvieron rendimientos similares a los que se logran con la fertilización mineral a $50 \%$ de la dosis recomendada al emplear gallinaza o biofertilizantes a base de micorrizas vesículo arbusculares; otros estudios indican que la gallinaza o las micorrizas versículo arbusculares (Díaz et al. 2016), así como la harina de pescado (Monares-Gallardo et al. 2012), también pueden sustituir la fertilización mineral.

Sin embargo, el uso de los fertilizantes minerales es indispensable, debido a la baja fertilidad de la mayoría de los suelos, por lo que éstos son necesarios para obtener altos rendimientos y calidad (FAO 2002); de hecho, se considera que más de $50 \%$ de los alimentos producidos mundialmente se atribuyen a la aplicación de este tipo de fertilizantes (Erisman et al. 2008; Stewart et al. 2005). Desafortunadamente, un problema frecuente es la baja eficiencia en el uso de los fertilizantes minerales, en gran medida debido a malas prácticas por parte de los productores. El manejo de la fertilización debe ser mejorado sustancialmente para reducir el impacto negativo sobre el medio ambiente, además de las implicaciones económicas, sociales y de salud pública (Mikkelsen 2011).

El fertirriego es una tecnología en la que se suplementan los fertilizantes mediante el riego en el momento y en el lugar en el que se requieren, lo cual permite obtener una eficiencia alta debido a la posibilidad de fraccionar las dosis de los fertilizantes en función de la tasa de absorción de los cultivos (Incrocci et al. 2017). Entre los nutrientes aplicados a través de fertirriego están el N, P y el potasio (K). El N es el más frecuentemente aplicado, pues, con excepción del sulfato de amonio, cualquier fuente de este elemento es altamente soluble y causa pocos problemas de precipitación y taponamiento de emisores; por su parte, en el caso del P, éste se prefiere aplicarlo en forma de ácido fosfórico o en otra forma altamente soluble (fosfato de potasio), para mantener un $\mathrm{pH}$ ácido y evitar precipitación con calcio (Ca) (Solaimalai et al. 2005). El K es el tercer elemento más frecuentemente aplicado $\mathrm{y}$, con excepción del sulfato de potasio, cuando se emplea en aguas de $\mathrm{pH}$ alcalino y altas en $\mathrm{Ca}$, sus formas son muy solubles (Solaimalai et al. 2005).

Aunque la aplicación de la tecnología del fertirriego tiene algunas desventajas, como son los altos costos de inversión, la probabilidad de que en épocas lluviosas se suministren los fertilizantes en una dosis menor a la demanda del cultivo, la necesidad de mano de obra especializada y el riesgo de obstrucción de los emisores (Incrocci et al. 2017), sus ventajas son mayores, al grado de que, a escala mundial, el área total cultivada con sistemas de fertirriego se ha incrementado 10 veces entre 1986 y 2015 (Reinders 2007; ICID 2016). 
Entre las ventajas del fertirriego destaca el hecho de que los nutrientes se aplican en la zona húmeda de la raíz, justo donde pueden ser absorbidos por los cultivos (Incrocci et al. 2017), lo que permite reducir las pérdidas por lixiviación. Estas pérdidas se calculan considerando el suministro de nutrientes residuales en el suelo, además del cálculo de los requerimientos de nutrientes suplementarios, así como los nutrientes acumulados por las plantas en una superficie determinada (Mikkelsen 2011). Solaimalai et al. (2005) señalan que las pérdidas por lixiviación en fertilizantes aplicados vía fertirriego llegan a ser de $10 \%$, comparado con $50 \%$ de pérdidas en aquellos que se aplican por los métodos convencionales. El objetivo de este estudio fue determinar si la cantidad de fertilizantes minerales aplicados al cultivo de calabacita puede ser reducida en comparación con la dosis recomendada, sin efectos negativos en el rendimiento de fruto y el crecimiento de la planta al fraccionar la aplicación de los mismos a través de un sistema de fertirriego.

\section{Materiales y MÉtodos}

\section{Ubicación del experimento y material vegetal}

El estudio se llevó a cabo en el Campus Buenavista de la Universidad Autónoma Agraria Antonio Narro, ubicado en el sur de la ciudad de Saltillo, Coahuila

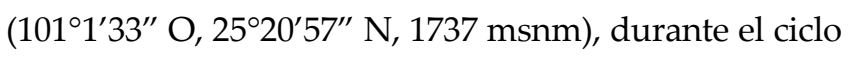
primavera-verano de 2016. El clima se considera desértico-semicálido, con una temperatura promedio de $18.8^{\circ} \mathrm{C}$ y una precipitación anual de entre 200 y 300 mm.

\section{Manejo del cultivo}

El suelo del sitio experimental tiene una textura de franco a franco-arcilloso, con una densidad aparente de $1.09 \mathrm{~g} \mathrm{cc}^{-1}, 40 \%$ de carbonatos totales, $2.20 \%$ de materia orgánica, conductividad eléctrica de $1.32 \mathrm{dS}$ $\mathrm{m}^{-1} \mathrm{y}$ un $\mathrm{pH}$ de 8.2. La preparación del suelo incluyó un barbecho profundo y un paso de rastra, para posteriormente formar las camas de siembra de $12 \mathrm{~m}$ de longitud, $1 \mathrm{~m}$ de ancho y una altura de $30 \mathrm{~cm}$, separadas entre sí por una distancia de $1.0 \mathrm{~m}$. El sistema de fertirriego consistió en un sistema de riego por goteo con cintillas con goteros autocompensados cada $0.3 \mathrm{~m}$ y con un gasto de $4 \mathrm{~L} \mathrm{~h}^{-1}$.

La siembra se efectuó de manera directa, a una profundidad de $5 \mathrm{~cm}$, con una semilla por mata y a una hilera por cama, con distancia de $1 \mathrm{~m}$ entre plantas. Las semillas fueron del cv. Grey Zucchini (Hydro Environment, Tlalnepantla, Estado de México); este cultivar es de polinización abierta y con frutos de color verde grisáceo de producción precoz. La densidad de población final fue de 10,000 plantas ha ${ }^{-1}$. Seis días después de la siembra se comenzó la aplicación de los fertilizantes de acuerdo con los tratamientos establecidos.

\section{Tratamientos}

Se estudiaron dos tratamientos de fertilización fraccionada vía fertirriego más un testigo; se aplicó una dosis de 120-33.6-174.3 $\mathrm{kg} \mathrm{ha}^{-1}$ de NPK en el cultivo de calabacita, indicado por Castellanos (2004). En el tratamiento testigo, en la siembra, se proporcionó 50\% del N y K y $100 \%$ del P, mientas que el restante $50 \%$ del $\mathrm{N}$ y K se aplicó 30 días después de la siembra.

Los tratamientos consistieron en reducir, con respecto al testigo, a $62.5 \%\left(75-20.8-108.1 \mathrm{~kg} \mathrm{ha}^{-1}\right)$ y a $50 \%\left(60-16.8-87.2 \mathrm{~kg} \mathrm{ha}^{-1}\right)$ las dosis de fertilización de $\mathrm{N}-\mathrm{P}-\mathrm{K}$, misma que se hizo por fertirriego en forma fraccionada. Para fraccionar la fertilización, 15 días después de la siembra se aplicó $16 \%$ del total de la dosis de fertilización, mientras que en el periodo de los días 16 al 30, del 31 al 45 y del 46 al 60, se aplicó 18\%, 34\% y $32 \%$ del total correspondiente a cada tratamiento. Las fuentes de fertilizante utilizados fueron: nitrato de amonio, fosfato monopotásico y cloruro de potasio. El sistema de riego por goteo permitió dosificar $4 \mathrm{~L} \mathrm{~h}^{-1}$ a una presión de 12 libras por pulgada cuadrada. La frecuencia de riego se determinó mediante tensiómetros colocados en diferentes puntos del área experimental a una profundidad de $25 \mathrm{~cm}$.

\section{Variables respuesta}

Las variables agronómicas evaluadas fueron: rendimiento total por planta, longitud y diámetro de fruto, peso seco de tallos y hojas, número de hojas y longitud del peciolo. Para evaluar el rendimiento, la cosecha se efectuó diariamente, cuando los frutos 
mostraban madurez comercial en dos plantas centrales por repetición, para eliminar el efecto de orilla. La cosecha se llevó a cabo entre las 7 y 8 am, con ayuda de una navaja y el punto de madurez se consideró cuando los frutos tenían aproximadamente $15 \mathrm{~cm}$ de longitud y mostraban marchitez de la corola. El primer y último corte se realizó a los 41 y 71 días después de la siembra, respectivamente.

Al finalizar el estudio, se muestreó la parte aérea de las plantas, la cual se lavó con agua destilada y se secó por $72 \mathrm{~h}$ en una estufa (Thermo Scientific Linderberg LB305565G $^{\circledR}$ Gravity Oven, Massachusetts, Estados Unidos), a una temperatura de $72^{\circ} \mathrm{C}$. Una vez secas, las plantas se molieron en un molino (Tekmar ${ }^{\circledR}$ modelo A-10, Cole-Parmer Instrument, Chicago, Estados Unidos), en el que se introducía el material vegetal, mediante el empleo de guantes de látex; para evitar contaminación, entre muestras, el molino se limpiaba con una aspiradora. Las muestras fueron molidas hasta alcanzar un tamaño menor a un filtro de 40 mallas.

El $\mathrm{N}$ se determinó con el procedimiento de semimicro Kjeldahl (Bremner 1996). Para los elementos como el $\mathrm{P}, \mathrm{K}, \mathrm{Ca}$ y $\mathrm{Mg}$, las muestras secas se digestaron en $2 \mathrm{~mL}$ de una mezcla de $\mathrm{H}_{2} \mathrm{SO}_{4}$ y $\mathrm{HClO}_{4}$ y $1 \mathrm{ml}$ de $\mathrm{H}_{2} \mathrm{O}_{2}$. El contenido nutrimental se determinó mediante un espectrómetro de emisión de plasma acoplado inductivamente (ICP-AES 725 Series Agilent ${ }^{\circledR}$, Mulgrave, Victoria, Australia) (Soltanpour et al. 1996).

Considerando la concentración de N, P y K en la parte aérea, así como el peso seco correspondiente de cada planta, se hicieron los cálculos necesarios para determinar la eficiencia en la recuperación de los nutrientes aportados en la fertilización. La eficiencia de recuperación se expresó como el porcentaje del nutriente absorbido por la planta en relación con el total aportado en la fertilización al suelo o vía fertirriego (Chuan et al. 2016), de acuerdo con cada tratamiento. La eficiencia agronómica se calculó con base en los gramos de fruto fresco cosechado por kilogramo de nutrimento total aportado en la fertilización al suelo o vía fertirriego (Chuan et al. 2016).

\section{Análisis estadístico}

El experimento fue establecido con un diseño experimental en bloques al azar, con tres repeticiones, y la unidad experimental estuvo constituida por cuatro plantas. El análisis de datos se llevó a cabo mediante análisis de varianza y la prueba de comparación de medias de Duncan $(p \leq 0.05)$ se efectuó por medio del programa estadístico SAS ver. 9.0.

\section{Resultados Y Discusión}

El rendimiento de fruto no fue afectado en plantas fertirrigadas con $62.5 \%$ ni $50 \%$ de la dosis de fertilización, en comparación con las plantas del tratamiento testigo (Figura 1). Las características físicas del fruto tampoco fueron afectadas por la reducción en la dosis de fertilización cuando se aplicó en forma fraccionada en ninguno de los tratamientos con fertirriego (Cuadro 1).

En el conteo de las hojas y el peso seco foliar,

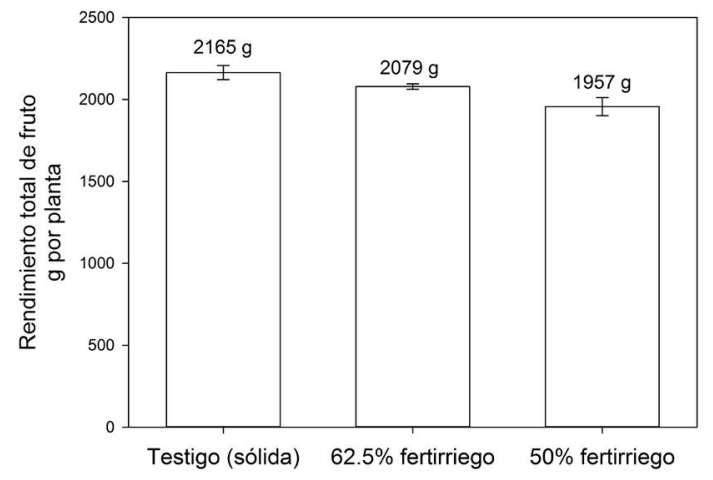

Figura 1. Rendimiento de fruto en plantas de calabacita (Cucurbita pepo cv Grey Zucchini) sometidas a dosis de N-P-K aplicada en forma sólida o fraccionada mediante sistema de fertirriego. El ANOVA no detectó efectos significativos $(p=0.403)$. Las líneas representan el error estándar de la media.

Cuadro 1. Longitud y diámetro de fruto (promedio \pm error estándar) de frutos cosechados de plantas de calabacita (Cucurbita pepo cv Grey Zucchini) sometidas a diferentes dosis de N-P-K aplicada en forma sólida o fraccionada mediante sistema de fertirriego.

\begin{tabular}{ccc}
\hline Tratamiento & $\begin{array}{c}\text { Longitud de fruto } \\
\mathrm{cm}\end{array}$ & $\begin{array}{c}\text { Diámetro de fruto } \\
\mathrm{cm}\end{array}$ \\
\hline $\begin{array}{c}100 \% \\
\text { (fertilización } \\
\text { sólida) } \\
62.5 \%\end{array}$ & $10.9 \pm 0.18$ & $3.8 \pm 0.08$ \\
$\begin{array}{c}\text { (fertirriego) } \\
50 \%\end{array}$ & $10.0 \pm 0.15$ & $3.3 \pm 0.11$ \\
(fertirriego) & $9.5 \pm 0.24$ & $3.5 \pm 0.03$ \\
\hline ANOVA & $p=0.147$ & $p=0.234$ \\
\hline
\end{tabular}


no se detectó efecto de los tratamientos (Cuadro 2), mientras que la longitud del peciolo fue reducida por las dosis bajas de fertilización, ya que éstos fueron $17 \%$ más cortos en las plantas que recibieron $50 \%$ de fertilización, en comparación con las plantas con el tratamiento testigo; por su parte, para aquellas que recibieron $62.5 \%$ de la fertilización, la reducción fue de $27 \%$ (Cuadro 2). El peso seco de tallos tampoco fue afectado por la reducción en la dosis de fertilización aplicada a través del fertirriego (Cuadro 2).

Los resultados obtenidos demuestran que, de 180 a $240 \mathrm{~kg} \mathrm{ha}^{-1}$. Lo anterior se explica por el hecho de que en ambos estudios los autores llevaron a cabo las aplicaciones de manera similar al manejo que se le proporcionó a las plantas del tratamiento testigo en el presente estudio.

Se ha definido que la aplicación fraccionada de los fertilizantes mediante el fertirriego es la mejor opción para el cultivo de calabacita (Mohammad 2004b), ya que aumenta el acceso a los nutrientes, debido a que el fertilizante se coloca en el lugar en donde la planta tiene más probabilidad de absorberlos y permite

Cuadro 2. Efecto de la dosis de N-P-K aplicada en forma sólida o fraccionada mediante sistema de fertirriego en hojas y tallos de plantas de calabacita (Cucurbita pepo cv Grey Zucchini) (promedio \pm error estándar).

\begin{tabular}{ccccc}
\hline Tratamiento & $\begin{array}{c}\text { Número de } \\
\text { hojas }\end{array}$ & Peso seco foliar & Longitud del peciolo & Peso seco de tallo \\
$\mathrm{cm}$ & $\mathrm{g}$ & $229.9 \pm 9.85$ & $53.7 \pm 0.76 \mathrm{a}$ & $34.6 \pm 4.59$ \\
\hline $100 \%$ (fertilización sólida) & $36.0 \pm 0.50$ & $144.4 \pm 14.74$ & $39.4 \pm 1.19 \mathrm{~b}$ & $28.2 \pm 1.58$ \\
$62.5 \%$ (fertirriego) & $40.0 \pm 2.93$ & $171.5 \pm 13.91$ & $44.4 \pm 1.34 \mathrm{~b}$ & $38.9 \pm 4.73$ \\
$50 \%$ (fertirriego) & $30.6 \pm 2.91$ & $p=0.105$ & $p=0.014$ & $p=0.408$ \\
\hline Anova & $p=0.254$ & &
\end{tabular}

Promedios con diferente letra indican diferencias significativas de acuerdo con la prueba de comparación múltiple de medias de Duncan ( $p \leq 0.05$ ).

mediante la aplicación fraccionada de los fertilizantes a través del fertirriego, es posible disminuir la dosis requerida por el cultivo de la calabacita hasta en $50 \%$ sin efectos en el rendimiento ni calidad de los frutos, por lo que aplicar los fertilizantes en forma fraccionada permite a las plantas disponer de los nutrientes justo en el momento en el que la demanda es alta, lo que disminuye la pérdida de estos. Mohammad (2004a) señala que, comparado con plantas que recibieron una fertilización convencional equivalente a $100 \mathrm{mg}$ $\mathrm{L}^{-1}$ de $\mathrm{N}$, la calabacita puede producir un rendimiento y crecimiento similar, a pesar de reducir la concentración de la solución de fertirriego hasta $50 \mathrm{mg}$ $\mathrm{L}^{-1}$ de $\mathrm{N}$ fertilizante, si esto se lleva a cabo a través del fertirriego, incluso hasta puede superar el rendimiento si la solución de fertirriego contiene $100 \mathrm{mg} \mathrm{L}^{-1}$ de N.

Sin embargo, los resultados del presente estudio contrastan con los reportados por Sedano-Castro et al. (2011), quienes indican que el rendimiento en calabacita es mayor, con dosis de 240 y $330 \mathrm{~kg} \mathrm{ha}^{-1}$ de $\mathrm{N}$, en comparación con $150 \mathrm{~kg} \mathrm{ha}^{-1}$. Lo mismo ocurre con los reportados por Kolota y Slociak (2006), quienes indican que el mayor rendimiento en esta especie se presenta con fertilizaciones nitrogenadas aplicar la dosis que demanda el cultivo (Mohammad 2004a), de acuerdo con la etapa fenológica, si para ello se siguen los modelos de extracción (Mohammad 2004a; Rodas-Gaitán et al. 2012).

La reducción en la dosis de fertilización y su aplicación fraccionada no afectó la concentración de $\mathrm{N}, \mathrm{P}, \mathrm{Ca}, \mathrm{y} \mathrm{Mg}$ en los tejidos de la parte aérea de las plantas (Cuadro 3); sin embargo, la concentración de $\mathrm{K}$ fue significativamente más baja cuando se redujo la fertilización a $50 \%$ de la dosis, aunque sin afectar el rendimiento de fruto (Figura 1). La menor concentración de $\mathrm{K}$ en plantas que recibieron $50 \%$ de fertilización puede deberse a que su requerimiento estuvo cubierto satisfactoriamente, a pesar de la dosis baja que recibieron, mientras que en los tratamientos en los que se elevó la dosis de $\mathrm{K}$ se presentó una mayor acumulación del nutriente, pero esto no se reflejó en un mayor crecimiento ni rendimiento; esta respuesta se ajusta al concepto de consumo de lujo de $\mathrm{K}$, el cual se ha reportado en varios cultivos (Basak et al. 2020; Hawkesford et al. 2012).

De acuerdo con Rodas-Gaitán et al. (2012), la calabacita demanda 6.75 g por planta de $\mathrm{N}$, mientras que el requerimiento de $\mathrm{P}$ y $\mathrm{K}$ es menor $(0.67$ y $1.37 \mathrm{~g}$ por 
Cuadro 3. Efecto de la dosis de N-P-K aplicada en forma sólida o fraccionada mediante sistema de fertirriego en la concentración nutrimental (mmol kg-1) (promedio \pm error estándar) de la parte aérea en plantas de calabacita (Cucurbita pepo cv Grey Zucchini).

\begin{tabular}{cccccc}
\hline Tratamiento & Nitrógeno & Fósforo & Potasio & Calcio & Magnesio \\
\hline $\begin{array}{c}100 \% \text { (fertilización } \\
\text { sólida) }\end{array}$ & $2468 \pm 122.0$ & $71.1 \pm 9.9$ & $341 \pm 28.2 \mathrm{ab}$ & $1951 \pm 57.8$ & $679 \pm 7.74$ \\
$62.5 \%$ (fertirriego) & $2733 \pm 49.7$ & $115.3 \pm 14.6$ & $383 \pm 46.3 \mathrm{a}$ & $1602 \pm 93.0$ & $644 \pm 51.8$ \\
$50 \%$ (fertirriego) & $2444 \pm 116.9$ & $90.8 \pm 6.54$ & $275 \pm 5.00 \mathrm{~b}$ & $1700 \pm 250.0$ & $601 \pm 73.5$ \\
\hline ANOVA & $p=0.286$ & $p=0.149$ & $p=0.049$ & $p=0.324$ & $p=0.486$ \\
\hline
\end{tabular}

Promedios con diferente letra indican diferencias significativas de acuerdo con la prueba de comparación múltiple de medias de Duncan ( $p \leq 0.05$ ).

planta, respectivamente). Esta demanda es aproximada a la observada en el presente estudio, ya que, con excepción del K, el $\mathrm{N}$ acumulado fue de 7.96, 6.56 y 6.53 g por planta, al recibir la dosis de fertilización completa y a $62.5 \%$ y $50 \%$, respectivamente, mientras que la demanda de $P$ fue de $0.51,0.62$ y 0.53 g por planta, y la de $\mathrm{K}$ fue de 0.36 , 0.27 y $0.21 \mathrm{~g}$ por planta.

A pesar de ser sometidas a una dosis de fertilización más baja, las plantas con aplicaciones fraccionadas a través del fertirriego mostraron una mayor eficiencia en la recuperación del $\mathrm{N}$ y $\mathrm{P}$, la cual se basa en la cantidad del elemento acumulado en la biomasa total, en comparación con las plantas a las cuales la fertilización se les aplicó en forma sólida y a $100 \%$ de la dosis (Figura 2). Con respecto al K, aunque se observa una tendencia similar, no se logró demostrar diferencias entre tratamientos (Figura 2).

En cuanto a la eficiencia agronómica, basada en el rendimiento, las plantas que recibieron la aplicación fraccionada de los fertilizantes tuvieron una mayor producción de fruto por cada unidad de N, P y K aplicado durante el ciclo del cultivo, de acuerdo con los tratamientos en estudio, por lo que superaron significativamente lo observado en plantas con una fertilización sólida del tratamiento testigo (Figura 3).

La mayor eficiencia observada en el presente estudio se debe a que los fertilizantes se aplicaron en forma fraccionada y en el momento de mayor demanda por parte del cultivo, con lo que se logra que las plantas dispongan del elemento cuando el requerimiento es mayor. El concepto " $4 \mathrm{R}$ " se refiere a la aplicación de nutrientes en la forma, fuente, lugar y tiempo correctos, lo que permite un manejo adecuado de los fertilizantes y contribuye a una agricultura sostenible (Bruulsema et al. 2009). Sin embargo, para el diseño de un programa de fertilización eficaz se requiere determinar con
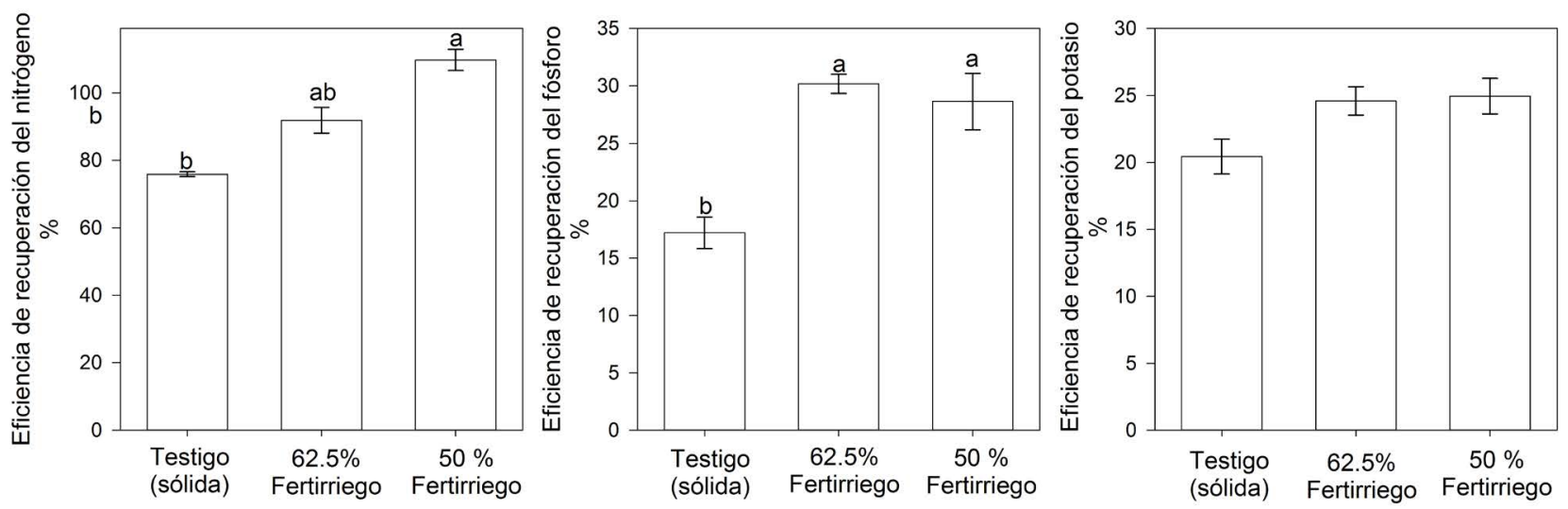

Figura 2. Eficiencia de recuperación nutrimental para nitrógeno (ERN), fósforo (ERP) y potasio (ERK), en plantas de calabacita (Cucurbita pepo cv Grey Zucchini) sometidas a dosis de N-P-K aplicadas en forma sólida o fraccionada, mediante sistema de fertirriego. El ANOVA detectó efectos significativos para ERN ( $p=0.042$ ) y ERP $(p=0.048)$, pero no para ERK $(p=0.497)$. Promedios con diferente letra indican diferencias significativas de acuerdo con la prueba de comparación múltiple de Duncan $(p \leq 0.05)$. Las líneas representan el error estándar de la media. 
precisión las cantidades de fertilizantes necesarios para complementar el suministro de nutrientes del suelo (Mattson y van Iersel 2011; Santos 2011; Roberts 2008). En el caso de calabacita, Rodas-Gaitán et al. (2012) reportaron que la absorción de los macronutrientes se acelera después del inicio de la floración, entre el día 20 y el 30 después de la siembra, y se presenta otro aumento en la tasa de acumulación posterior a los 50 días de la siembra; en esas etapas la fertilización debe aplicarse en mayor cantidad para cubrir las demandas del cultivo.

La mayor eficiencia de recuperación y eficiencia que las dosis de $\mathrm{N}$ aplicadas en este estudio fueron las adecuadas.

La menor eficiencia de recuperación en $\mathrm{P}$ y K puede deberse a la baja movilidad de estos elementos una vez que se depositan en el suelo gracias a la solución del fertirriego; se ha reportado que el $\mathrm{P}$ reacciona rápidamente con arcillas como montmorrilonita y ilita, o bien con los carbonatos, en suelos básicos (Sandal y Kapoor 2015), como el característico del sitio del presente estudio. Nuestros resultados coinciden con lo señalado por Mohammad (2004b), quien establece que el fertirriego es la mejor técnica de aplicación de los fertilizantes si éstos
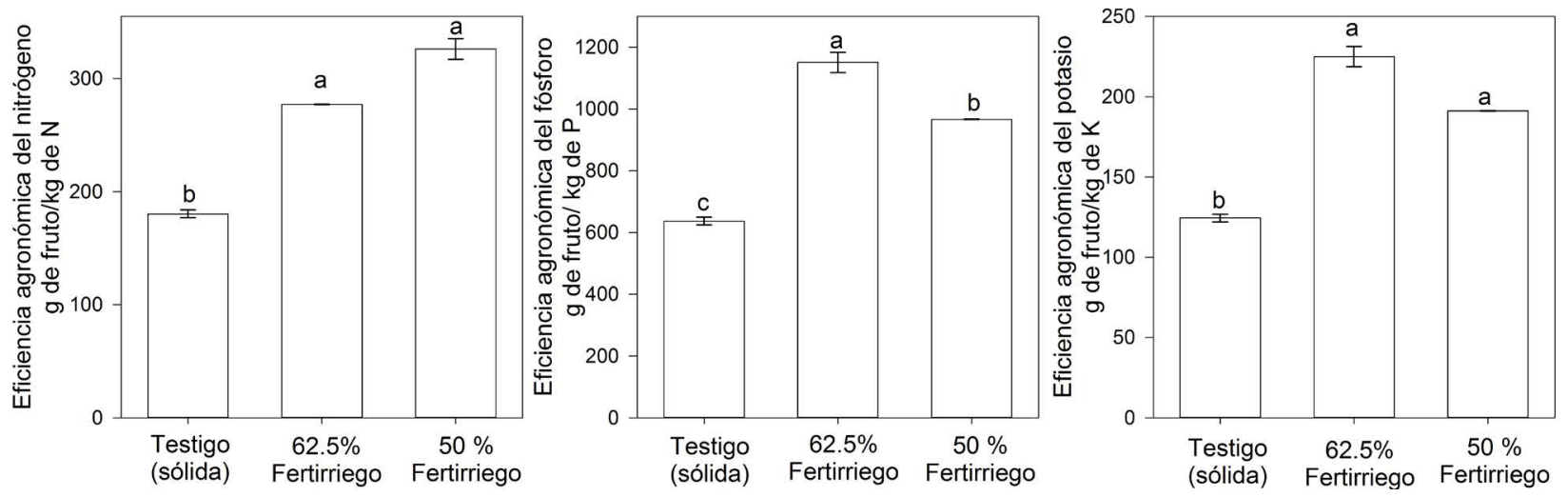

Figura 3. Eficiencia agronómica para nitrógeno (EAN), fósforo (EAP) y potasio (EAK) en plantas de calabacita (Cucurbita pepo cv Grey Zucchini) sometidas a dosis de N-P-K aplicada en forma sólida o diluida mediante sistema de fertirriego. El ANOVA detectó efectos significativos para EAN ( $p=0.003)$, EAP ( $p=0.004)$ y EAK ( $p=0.015)$. Promedios con diferente letra indican diferencias significativas de acuerdo con la prueba de comparación múltiple de Duncan ( $p \leq 0.05)$. Las líneas representan el error estándar de la media.

agronómica en plantas con dosis de fertilización reducida sugiere que la aplicación oportuna de los fertilizantes disminuyó sustancialmente las pérdidas por lixiviación; esto, a su vez, se reflejó en una mayor eficiencia en el uso de los mismos, así como en un menor daño al medio ambiente y una menor pérdida económica (Chuan et al. 2016).

Según Solaimalai et al. (2005), las pérdidas por lixiviación en fertilizantes aplicados vía fertirriego llegan a ser de $10 \%$, comparado con $50 \%$ de pérdidas en aquellos que se aplican por los métodos convencionales; en el presente estudio, se lograron obtener eficiencias en la recuperación del $\mathrm{N}$ de más de $90 \%$; en el caso del $\mathrm{P}$, aunque se logró duplicar de $17 \%$ hasta $30 \%$, la eficiencia es aún baja; finalmente, en el caso de $\mathrm{K}$, no se obtuvieron mejoras sustanciales en la eficiencia de recuperación (Figura 2). Esto sugiere que probablemente se puedan reducir aún más las dosis de $\mathrm{P}$ y $\mathrm{K}$, mientras se fraccionan y se aplican en mayor proporción cuando la demanda por parte del cultivo se incrementa.

\section{Conclusiones}

La fertilización fraccionada aplicada a través del fertirriego permite reducir la dosis recomendada de fertilización hasta en $50 \%$, sin afectar el rendimiento ni el crecimiento de las plantas; asimismo, esto estuvo relacionado con un aumento en la eficiencia de recuperación, pues, en comparación con la fertilización tradicional, ésta fue entre $21 \%$ y $45 \%$ mayor para el N y entre $67 \%$ y $76 \%$ para el $\mathrm{P}$, mientras que la eficiencia agronómica fue entre $53 \%$ y $80 \%$ para el $\mathrm{N}$, entre $52 \%$ y $81 \%$ para el $\mathrm{P}$, así como entre $54 \%$ y $80 \%$ para $\mathrm{K}$, comparado con la fertilización tradicional. 


\section{LITERATURA CITADA}

Alvarado-Carrillo M, Díaz-Franco A, Alejandro-Allende F. 2018. Gallinaza, micorriza arbuscular y fertilización química reducida en la productividad de calabacita y pepino. Revista Internacional de Contaminación Ambiental 34: 273-279. http://doi.org/10.20937/ RICA.2018.34.02.08

Apáez-Barrios P, Lara-Chávez MBN, Apáez-Barrios M, Raya-Montaño YA. 2019. Producción y rentabilidad de calabacita con aplicación de zeolita y fertilizante químico. Revista Mexicana de Ciencias Agrícolas 23: 211-221. http://doi.org/10.29312/remexca.v0i23.2022

Basak BB, Maity A, Biswas DR. 2020. Cycling of natural sources of phosphorus and potassium for environmental sustainability. In: Dontsova K, Balogh-Brunstad Z, Le Roux G, editors. Biogeochemical Cycles: Ecological Drivers and Environmental Impact. New Jersey, Wiley. P. 285-299.

Bremner JM. 1996. Total nitrogen. In: Sparks DL, editor. Methods of Soil Analysis. Part 2. Chemical Methods. American Society of Agronomy. Madison, Soil Science Society of America. P. 1085-1086.

Bruulsema T, Lemunyon J, Herz B. 2009. Know your fertilizer rights. Crops and Soils 42: 13-18.

Castellanos JZ. 2004. Manual de producción hortícola en invernaderos. INTAGRI. Celaya, México.

Chance III WO, Somda ZC, Mills HA. 1999. Effect of nitrogen form during the flowering period on zucchini squash growth and nutrient element uptake. Journal of Plant Nutrition 22: 597-607. http://doi. org/10.1080/01904169909365655

Chuan L, He P, Zhao T, Zheng H, Xu X. 2016. Agronomic characteristics related to grain yield and nutrient use efficiency for wheat production in China. PLoS ONE 11(9): e0162802. http://doi.org/10.1371/journal.pone.0162802

Díaz A, Alvarado M, Alejandro F, Ortiz FE. 2016. Crecimiento, nutrición y rendimiento de calabacita con fertilización biológica y mineral. Revista Internacional de Contaminación Ambiental 32: 445-453. http://doi. org/10.20937/RICA.2016.32.04.08

Erisman JW, Sutton MA, Galloway JN, Klimont Z, Winiwarter W. 2008. How a century of ammonia synthesis changed the world. Nature Geoscience 1: 636-639. http://doi.org/10.1038/ngeo325

[FAO] Food and Agriculture Organization. 2002. Los fertilizantes y su uso. Asociación Internacional de la Industria de los Fertilizantes. Roma, Italia.
Hawkesford M, Horst W, Kichey TMR, Schjørring JK, Møller IS, White P. 2012. Functions of macronutrients. In: Marschner P, editor. Marschner's Mineral Nutrition of Higher Plants. San Diego, Elsevier. P. 135-189.

Hendricks GS, Shukla S, Roka FM, Sishodia RP, Obreza TA, Hochmuth GJ, Colee J. 2019. Economic and environmental consequences of overfertilization under extreme weather conditions. Journal of Soil and Water Conservation 74: 160-171. http://doi.org/10.2489/ jswc.74.2.160

[ICID] International Commission on Irrigation and Drainage. 2016. Agricultural Water Management for Sustainable Rural Development: Annual Report 20152016. International Commission on Irrigation and Drainage. New Delhi, India.

Incrocci L, Massa D, Pardossi A. 2017. New trends in the fertigation management of irrigated vegetable crops. Horticulturae 3: 37. http://doi.org/10.3390/ horticulturae 3020037

Kolota E, Slociak A. 2006. Nitrogen fertilization of zucchini harvested at different stages of fruit development. Acta Horticulturae 700: 121-124. http://doi.org/10.17660/ ActaHortic.2006.700.17

Mattson NS, van Iersel MW. 2011. Application of the " $4 \mathrm{R}$ " nutrient stewardship concept to horticultural crops: Applying nutrients at the "right time". HortTechnology 11: 667-673. http://doi.org/10.21273/ HORTTECH.21.6.667

Mikkelsen RL. 2011. The " $4 R$ " nutrient stewardship framework for horticulture. HortTechnology 21: 658-662. http://doi.org/10.21273/HORTTECH.21.6.658

Mohammad MJ. 2004a. Squash yield, nutrient content and soil fertility parameters in response to methods of fertilizer application and rates of nitrogen fertigation. Nutrient Cycling in Agroecosystems 68: 99-108. https:// doi.org/10.1023/B:FRES.0000019036.64212.9c

Mohammad MJ. 2004b. Utilization of applied fertilizer nitrogen and irrigation water by drip-fertigated squash as determined by nuclear and traditional techniques. Nutrient Cycling in Agroecosystems 68: 1-11. https:// doi.org/10.1023/B:FRES.0000012229.61906.6c

Monares-Gallardo I, Ceja-Torres LF, EscaleraGallardo C, Vázquez-Gálvez G, Ochoa-Estrada S. 2012. Tamaño de partícula y tiempo de aplicación pre-siembra de harina de pescado (Plecostomus spp.) en producción de calabacita. Terra Latinoamericana 30: 147-155. 
Reinders FB. 2007. Micro-irrigation: World overview on technology and utilization. Proceedings of the 7th International Micro-Irrigation Congress. Kuala Lumpur, Malaysia

Roberts TL. 2008. Improving nutrient use efficiency. Journal of Agriculture and Forestry 3: 177-182.

Rodas-Gaitán HA, Rodríguez-Fuentes H, Ojeda-Zacarías MdelC, Vidales-Contreras JA, Luna-Maldonado AI. 2012. Curvas de absorción de macronutrientes en calabacita italiana (Cucurbita pepo L.). Revista Fitotecnia Mexicana 35 (Núm. Esp. 5): 57-60.

Sadeghpour A, Ketterings QM, Godwin GS, Czymmek KJ. 2017. Under- or over-application of nitrogen impact corn yield, quality, soil, and environment. Agronomy Journal 109: 343-353. http://doi.org/10.2134/agronj2016.06.0355

Sandal SK, Kapoor R. 2015. Fertigation technology for enhancing nutrient use and crop productivity: An overview. Himachal Journal of Agricultural Research 41: 114-121.

Santos BM. 2011. Selecting the right nutrient rate: Basis for managing fertilization programs. HortTechnology 21: 683-685. http://doi.org/10.21273/HORTTECH.21.6.683

Sedano-Castro G, González-Hernández VA, Saucedo-Veloz C, Soto-Hernández M, Sandoval-Villa M, CarrilloSalazar JA. 2011. Rendimiento y calidad de frutos de calabacita con altas dosis de $\mathrm{N}$ y K. Terra Latinoamericana 29: 133-142.

[SIAP] Servicio de Información Agroalimentaria y Pesquera. 2019. Panorama agroalimentario 2019. Secretaría de Agricultura y Desarrollo Rural. México.

Solaimalai A, Baskar M, Sadasakthi A, Subburamu K. 2005. Fertigation in high value crops. Agricultural Reviews 26: 1-13.

Soltanpour PN, Johnson GW, Workman SM, Jones JB, Miller RO. 1996. Inductively coupled plasma emission spectrometry and inductively coupled plasma mass spectrometry. In: Sparks DL, editor. Methods of Soil Analysis. Part 3. Chemical Methods. Madison, Soil Science Society of North America. P. 91-139.

Stewart WM, Dibb DW, Johnston AE, Smyth TJ. 2005. The contribution of commercial fertilizer nutrients to food production. Agronomy Journal 97: 1-6. http://doi. org/10.2134/agronj2005.0001 